\title{
Effects of Medium-chain Triglycerides Administration in Chemically-induced Carcinogenesis in Mice
}

\author{
HIROYUKI WAKANA, HIROSHI KONO, HISATAKA FUKUSHIMA, YUUKI NAKATA, YOSHIHIRO AKAZAWA, \\ SUGURU MARUYAMA, KOTARO HAGIO, HIDEKI FUJII and DAISUKE ICHIKAWA
}

First Department of Surgery, Faculty of Medicine, University of Yamanashi, Yamanashi, Japan

\begin{abstract}
Aim: The aim of this study was to investigate the effects of medium-chain triglycerides (MCTs) on chemicallyinduced hepatic carcinogenesis (HCC) in mice. Materials and Methods: In a first set of experiments, mice were treated with diethylnitrosoamine intraperitoneally at two weeks of age. They were fed chow containing MCT or a normal chow diet and sacrificed after 28 weeks. Incidence of hepatic tumor was compared between the two groups. Expression of oxidative stress, and inflammatory cytokines and chemokines in liver tissues were examined. In a second set of experiments, the histopathological findings of the intraperitoneal adipose tissue were assessed, and expression of adipocytokines in the fat tissue was measured. In a third set of experiments, plasma $\beta$-hydroxybutyrate $(H B)$ concentration was measured in both animals fed chow containing MCT and a normal chow diet. Mouse HCC cells were co-cultured with $\beta-H B$, and the numbers of tumor cells were counted at days 3 and 7. Results: In the first set of experiments, the tumor count observed in the control group was significantly blunted in the MCT group. Maximum tumor diameter also decreased in the MCT group compared to the control group. The expression of inflammatory cytokines and chemokines was significantly decreased by MCT. Furthermore, expression of 4hydroxynonenal was lower in the MCT group compared to the control group. In the second set of experiments, hypertrophy of the adipocytes was suppressed, and the concentration of adiponectin and leptin in the adipose tissue decreased by $M C T$. In the third set of experiments, plasma $\beta$-HB concentration increased in the MCT group as expected. $\beta-H B$ significantly inhibited the proliferation of HCC cells.
\end{abstract}

Correspondence to: Hiroshi Kono, First Department of Surgery, Faculty of Medicine, University of Yamanashi, 1110 Shimokato, Chuo, Yamanashi 409-3898, Japan. Tel: +81 0552737390, Fax: +81 0552736751,e-mail: hkouno@yamanashi.ac.jp

Key Words: Medium-chain triglycerides, anti-inflammatory effect, antioxidant action, adipocytokine, diethylnitrosamine, hepatocellular carcinoma, ketone body.
Conclusion: MCT administration markedly suppresses the incidence of chemically-induced HCC by inhibition of inflammation and increase of ketone bodies.

Nutrition is a key factor in carcinogenesis (1). Medium-chain triglycerides (MCTs) are easily absorbed from the small intestine without micelle formation by bile and transported to the liver via the portal vein. They are beta-oxidized in hepatocytes more rapidly than other fatty acids as an energy source (2), and have therefore been used in the medical field as a fatty preparation for postoperative nutrition or malnutrition. It is also known that MCTs produce ketone bodies in large quantities in the liver under conditions of low carbohydrate intake (3).

Prolonged inflammation, associated with viral infection or alcoholic/nonalcoholic steatohepatitis, increases oxidative stress, which is thought to be closely involved in the mechanism of hepatocellular carcinogenesis (4). It was previously reported from this laboratory that MCTs have an anti-inflammatory effect on activated macrophages $(5,6)$. In addition, MCTs also enhance the expression of $\operatorname{IgA}$ in the gastrointestinal tract (7).

In this study, we investigated the effects of MCT on chemically-induced hepatocellular carcinoma caused by intraperitoneal administration of diethylnitrosoamine (DEN), and the effects of MCTs on intraperitoneal fatty tissues and their associated adipocytokine expression. We also assessed the direct effect on cancer cell proliferation of a ketone body, $\beta$ hydroxybutyrate (HB), which is one of the metabolites of MCTs.

\section{Materials and Methods}

Animals. Male $\mathrm{C} 3 \mathrm{H} / \mathrm{HeN}$ Slc were housed in a clean, temperaturecontrolled environment with a 12-h light-dark cycle and were given free access to a regular laboratory water and powder diet for several days. All animals received humane care, and the study protocols were approved by the Committee of Laboratory Animals at the University of Yamanashi, in accordance with institutional guidelines.

Treatment and collection of samples. In a chemical carcinogenesis model, 14-day-old Male C3H/HeN Slc mice were treated with DEN 


\section{A: Control}
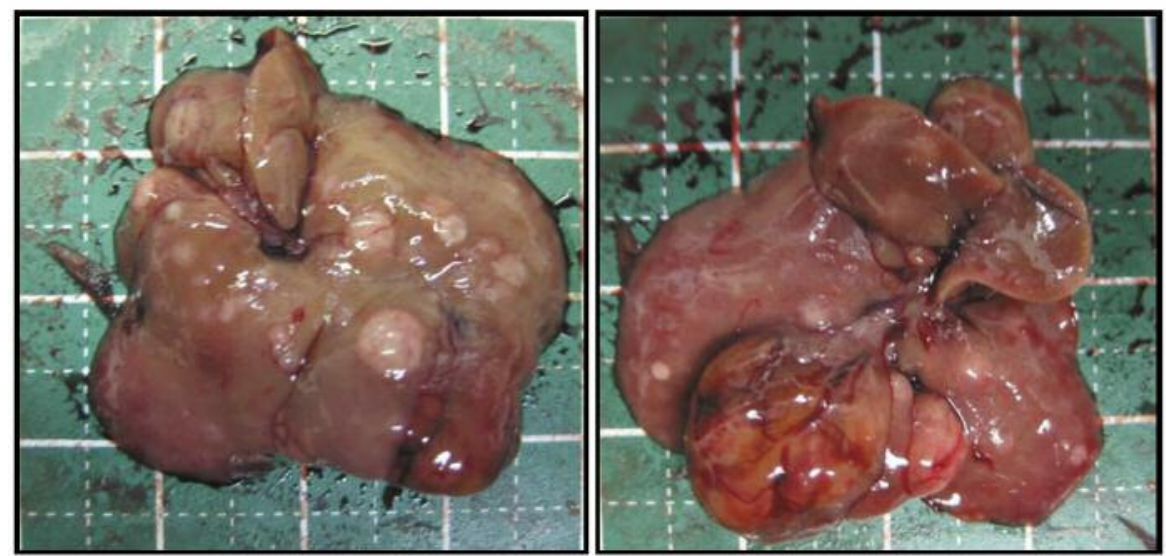

\section{B: MCT}
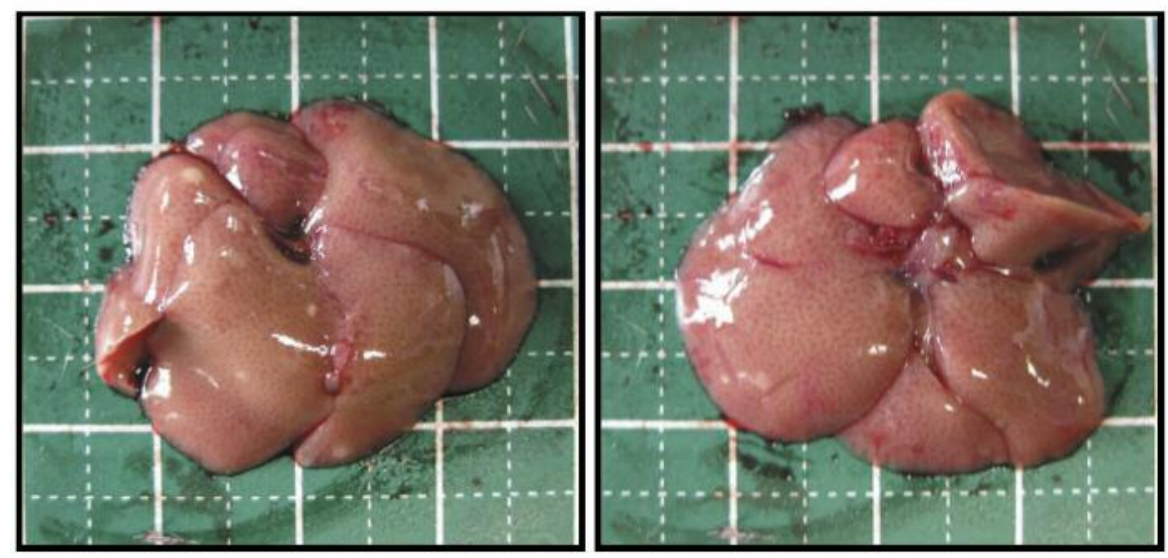

C

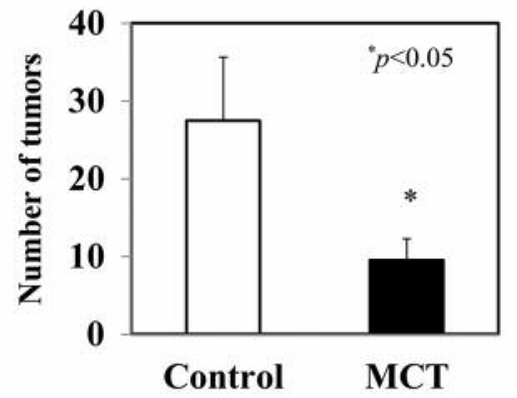

D

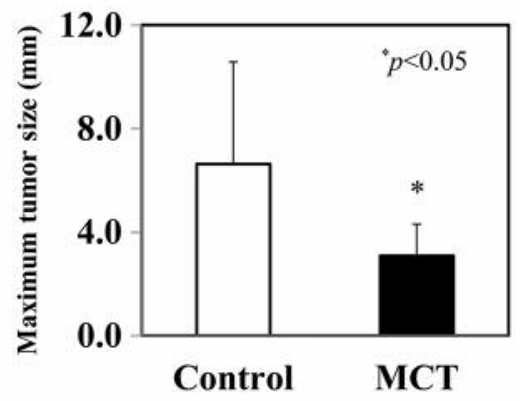

Figure 1. Tumor incidence and growth. A: control group, and B: MCT group. The numbers of tumors $(D)$ and maximum tumor size (E) are shown. Data represent means $\pm S E M .{ }^{*} p<0.05$ compared to the control group by the unpaired Student's $t$-test. MCT: Medium-chain triglycerides.

( $20 \mathrm{mg} / \mathrm{kg}$ of bodyweight, single intraperitoneal injection). These mice were anesthetized and killed 28 weeks later.

Animal diets. After administration of DEN, mice were divided into two groups. Each was fed with normal diet (corn oil: $\omega=6$ linoleic acid; Control diet group) and MCT $(\mathrm{C}=8$, octanoate; MCT diet group) added diet. To avoid essential fatty acid deficiency, corn oil ( $2 \%$ of total fat) was supplemented in the MCT diets. These powder diets were produced based on AIN93M from Oriental Yeast (Tokyo, Japan). The diets were given freely throughout the experimental periods, and body weights were recorded once a week. There were no significant differences in diet intake between the two groups throughout all experiments (60 g/week/mouse).

Measurement of plasma ketone bodies. Blood samples were collected via the inferior vena cava at the designated time points after 28 weeks of the DEN treatment from the Control diet group and the MCT diet group. The samples were centrifuged at $3,000 \mathrm{rpm}$ for $10 \mathrm{~min}$ at $4^{\circ} \mathrm{C}$, and plasma was stored at $-80^{\circ} \mathrm{C}$. Plasma $\beta$-HB levels were measured to assess increase and decrease of ketone bodies by MCT administration using a fluorometric Assay kit (Cayman Chemical Company, Ann Arbor, MI, USA) according to the manufacturer's instructions.

Immunohistochemistry for 4-HNE. Briefly, formalin-fixed, paraffinembedded tissue specimens were cut into 4- $\mu$ m sections. Each section was mounted on a silane-coated glass slide, deparaffinized, and incubated in antigen retrieval solution for $15 \mathrm{~min}$ at $121^{\circ} \mathrm{C}$ using Dako REAL Target Retrieval Solution (Dako, Carpinteria, CA, USA). Endogenous peroxidases were quenched by incubation at room temperature in $0.3 \% \mathrm{H}_{2} \mathrm{O}_{2}$, followed by rinsing with PBS. Endogenous biotin was quenched using Dako Biotin Blocking System (Dako). Sections were blocked using 5\% normal blocking serum for $20 \mathrm{~min}$. Rabbit polyclonal to 4-Hne (1:100 Abcam, Cambridge, UK) was applied overnight at $4^{\circ} \mathrm{C}$. Immunoperoxidase staining was completed using a Vectastain $\mathrm{ABC}$ elite kit (Vector Laboratories, Burlingame, CA, USA) and diaminobentizine (DAB) as a chromogen. The number of positive cells against each antibody was assessed by microscopy in five random $(400 \times)$ fields for 

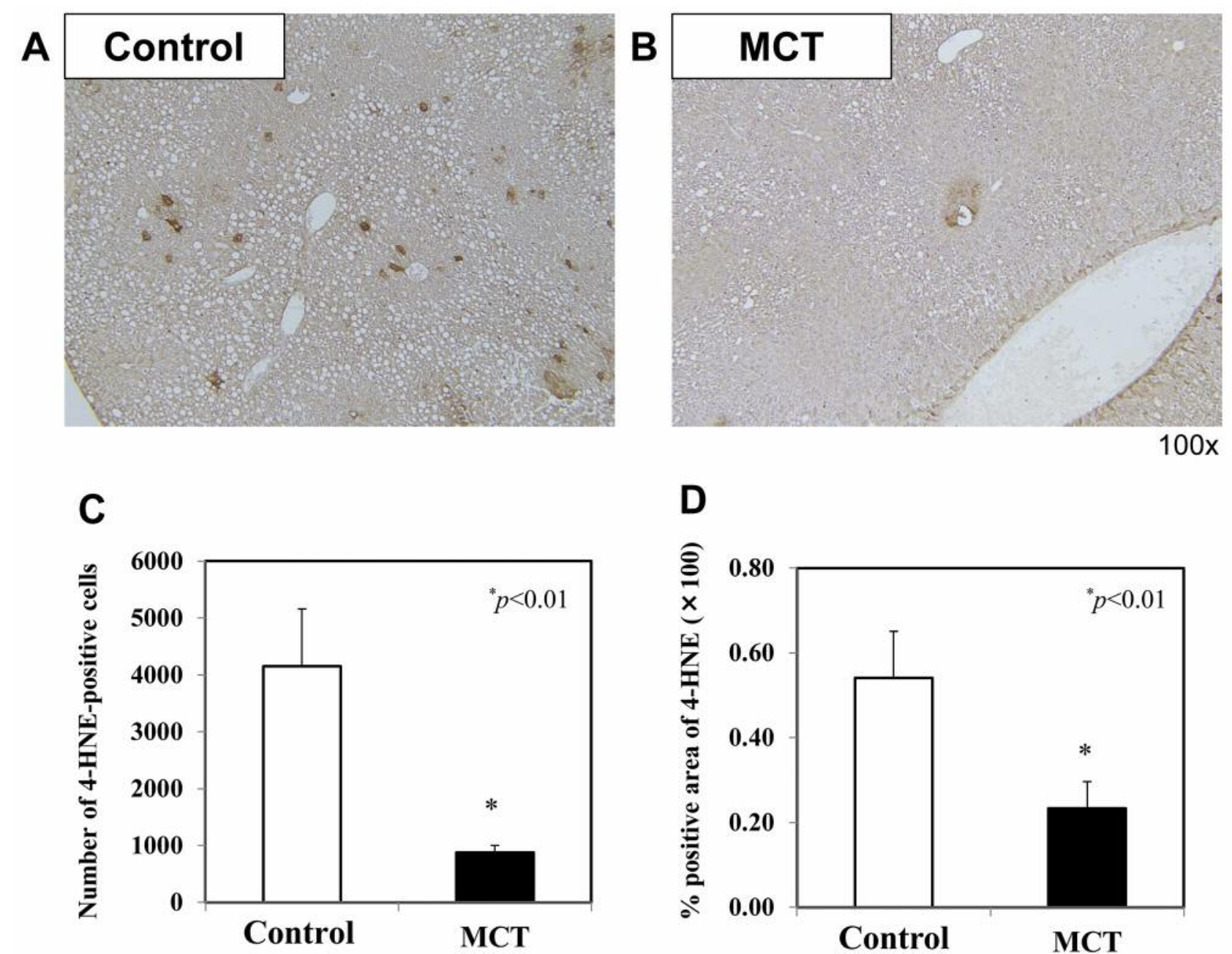

Figure 2. Immunohistochemistry for 4-HNE in the liver. Representative photomicrographs are shown (A: Control, and B: MCT). C: Number of 4HNE-positive cells, and D: \% positive area of 4-HNE. ${ }^{*} p<0.01$ compared to the control group by the unpaired Student's t-test.

calculation of the proliferation index. Histological samples were evaluated by one of the authors and by an external expert in rodent liver pathology. Quantitative analysis of the 4-HNE positive area was calculated from five different fields and indicated as a percentage of the total area of the field using the Image $\mathbf{J}$ software.

Measurement of inflammatory cytokines and chemokines by enzymelinked immunosorbent assay (ELISA). Liver tissue and intraperitoneal fat tissue were homogenized in cold phosphate-buffered saline using a Polytron-type homogenizer. The tissue homogenate was then centrifuged at $8,000 \mathrm{rpm}$ for $10 \mathrm{~min}$ at $4^{\circ} \mathrm{C}$ to obtain the supernatant. The total protein concentrations of the tissue supernatant and wholecell lysate were measured using a Bio-Rad protein assay kit (Bio-Rad Laboratories, Hercules, CA, USA) for calibration, and the protein concentrations of TNF- $\alpha$, IL-6, IL- $1 \beta$, IFN- $\gamma$, MCP-1, MIP-2, adiponectin and leptin in the tissue homogenate and culture media were determined using ELISA kits (R\&D Systems Inc., Minneapolis, MN, USA) according to the manufacturer's instructions.
Experiment 1. DEN was administered intraperitoneally to mice at two weeks after birth, and animals were divided into two groups: MCT $(\mathrm{C}=8$, octanoate)-added diet or normal diet (corn oil: $\omega=6$ linoleic acid). The mice were anesthetized and killed 28 weeks later. The number of tumors and maximum tumor diameter were compared between the two groups. Immunohistochemical staining was performed using collected livers and compared histologically. Expressions of inflammatory cytokines and chemokines in liver tissues were measured by ELISA.

Experiment 2. To investigate the effect of MCT on intraperitoneal fat, morphological and histopathological findings of the intraperitoneal adipose tissue were assessed. In addition, the number of adipocytes was measured in one high power field. Expression of adipocytokines (adiponectin and leptin) in extracts of the adipose tissues was compared and examined using ELISA.

Experiment 3 . The ketone body $(\beta-\mathrm{HB})$ concentration in mouse plasma was measured using a fluorometric assay kit (Cayman 

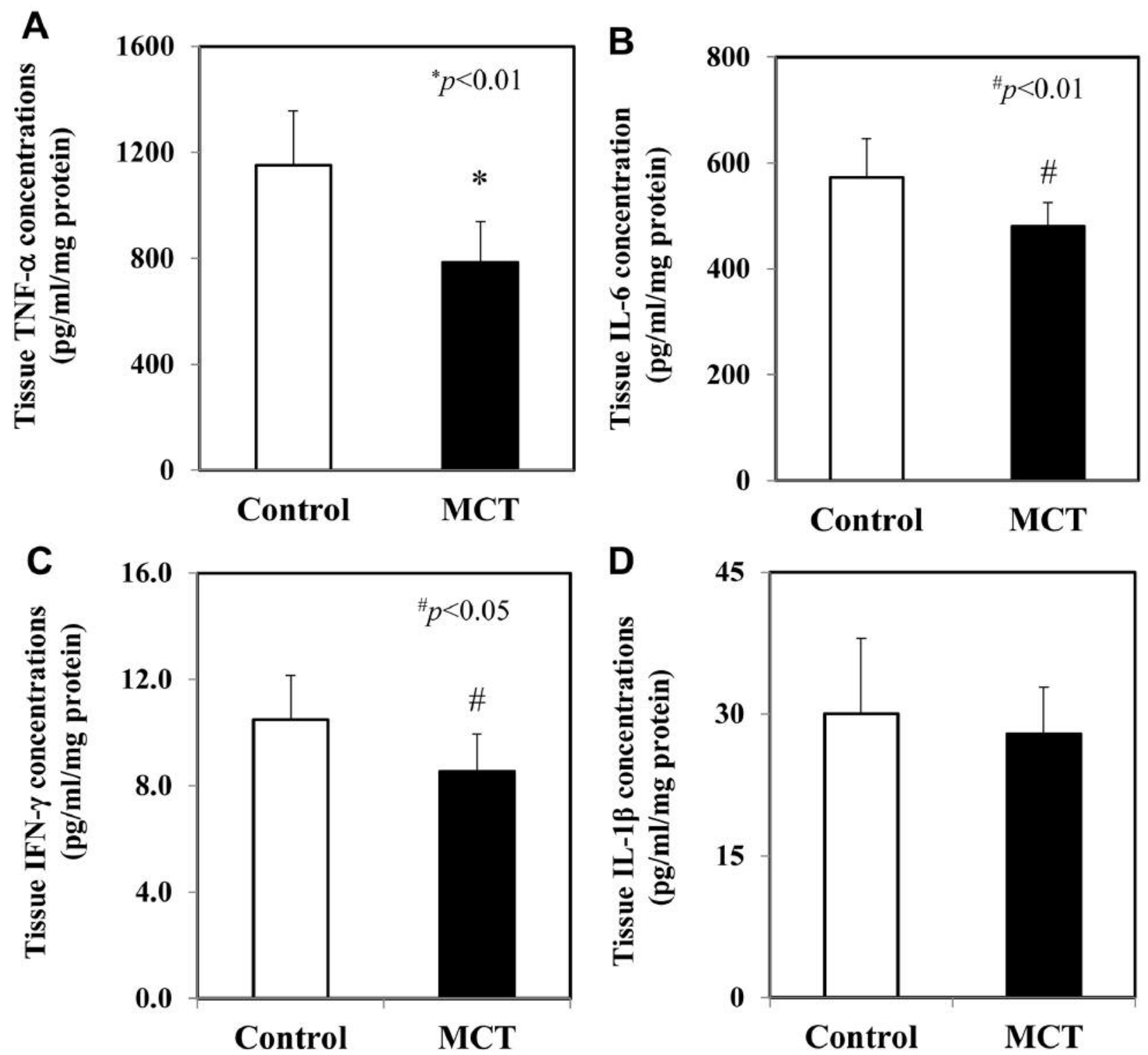

Figure 3. Expressions of inflammatory cytokines in liver tissues. A: TNF- $\alpha, B: I L-6, C: I N F-\gamma$, and D: IL-1 $\beta$. Data represent the mean \pm SEM. * $<<0.01$ compared with the control group, and \#p<0.05 compared with the control group by the unpaired Student's t-test. MCT: Medium-chain triglycerides.

Chemical Company) according to the manufacturer's instructions. To evaluate the effects of ketone bodies on tumor growth, mouse hepatocellular carcinoma cells (MH134) were co-cultured with $\beta$ HB. They were adjusted to density of $1.0 \times 10^{7} \mathrm{cell} / \mathrm{ml}$ and viability of $97 \%$, and $\beta-\mathrm{HB}$ was added at various concentrations $(0,200$, and $2,000 \mu \mathrm{g} / \mathrm{ml}$; normal blood $\beta$-HB reference range in humans is from 2 to $28 \mu \mathrm{g} / \mathrm{ml}$ ) into media, and incubated at $37^{\circ} \mathrm{C}$. On days 3 and 7 the numbers of cells were counted using an automated cell counter (Invitrogen CountessTM, Waltham, MA, USA).

Statistical analysis. Data are expressed as mean \pm SEM. Student's $t$ test was used for the determination of significance as appropriate. $p<0.05$ was considered significant.

\section{Results}

Experiment 1. Numerous hepatic tumors were observed macroscopically in the control group (Figure 1). The number of tumors was significantly blunted in the MCT groups compared with the control group. Furthermore, histopathological findings also showed that the average tumor sizes in the maximally-cleaved plane image were also significantly lower in the MCT groups compared with the control group (Figure 1), suggesting that the incidence of hepatic tumors and tumor growth were inhibited by MCT. 
A

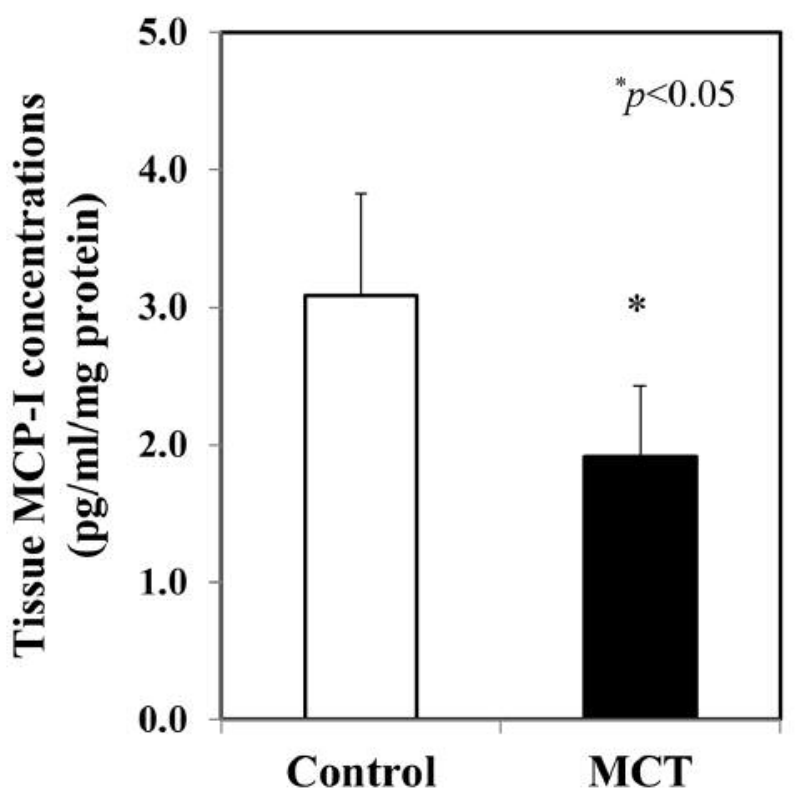

B

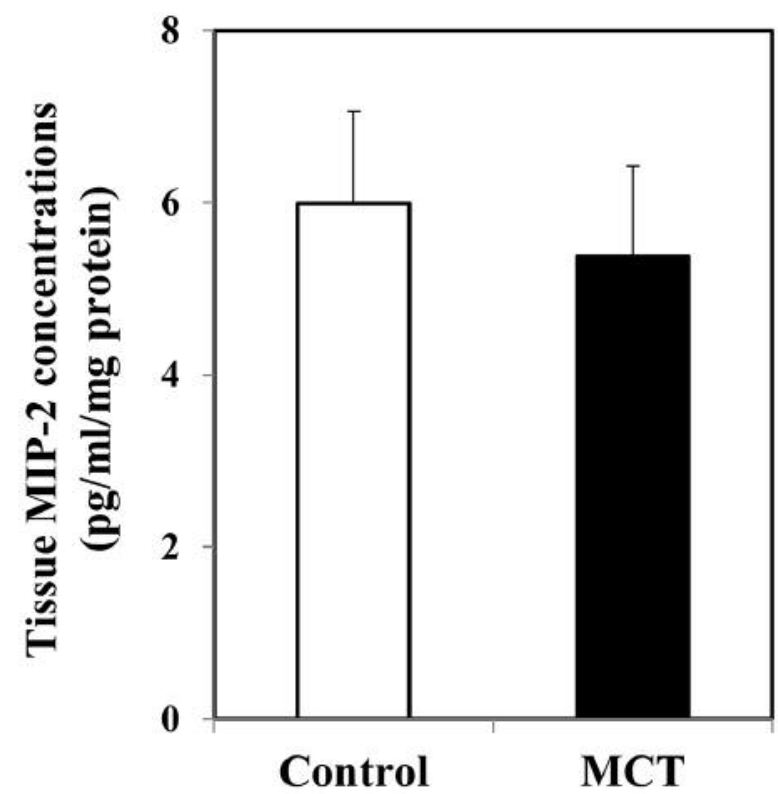

Figure 4. Expressions of chemokines in liver tissues. A: MCP-1, and B: MIP-2. Data represent the mean \pm SEM. *p $<0.01$ compared to the control group by the unpaired Student's t-test. MCT: Medium-chain triglycerides.

In 4-HNE immunohistochemical staining, positive hepatocytes increased in the control group after DEN treatment. This increase was significantly blunted in the MCT group (Figure 2), suggesting that lipid peroxidation was suppressed by MCT.

Expression of the inflammatory cytokines TNF- $\alpha$, IL-6, IL- $1 \beta$, and IFN- $\gamma$ increased in the control group after DEN treatment (Figure 3). Values were significantly lower in the MCT group compared with the control group. Similarly, the expressions of chemokines MCP-1 and MIP-2 increased in the control group after DEN treatment. Importantly, values were also significantly lower in the MCT group (Figure 4). Thus, MCTs suppressed the expression of inflammatory cytokines and chemokines in the liver tissues.

Experiment 2. Steady body weight gains were observed in the control group (Figure 5). The liver weight/body weight ratio was significantly suppressed in the MCT group compared to the control group (data not shown).

In histopathological findings of the intra-abdominal fat tissue, hypertrophy of adipocytes was observed in the control group. In contrast, hypertrophy was significantly suppressed in the MCT group (Figure 5).

Expression of adipocytokines including adiponectin and leptin produced by adipose tissue was significantly higher in the MCT group compared with the control group (Figure 6).
Experiment 3. The plasma ketone body $(\beta-\mathrm{HB})$ concentration was minimal in the control group (Figure 7), while a significant increase was observed in the MCT group, as expected.

Mouse HCC cells (MH134 cells) proliferated in time dependent manner in the absence of $\beta$-HB in media. In contrast, $\beta$-HB inhibited cell proliferation in a dose dependent manner (Figure 7). On days 3 and 7, there were significant differences in the number of MH134 cells.

\section{Discussion}

MCTs suppressed the number of hepatic tumors induced by DEN, indicating that MCTs inhibit the incidence of hepatic oncogenesis or tumor growth (Figure 1). It was reported from this laboratory that MCTs have anti-inflammatory effects (6). MCTs suppressed the expression of inflammatory cytokines and chemokines (Figures 4 and 5). After DEN administration, oxidative stress increased (8). Since MCTs suppressed expression of 4-HNE after DEN administration (Figure 3), MCTs inhibit oxidative stress derived from lipid peroxidation, leading to the inhibition of oncogenesis and tumor growth after DEN administration.

Since MCTs metabolize as an energy source more rapidly than other fatty acids (3), they inhibit fat accumulation in the liver. Indeed, body weight gains and the extent of fatty liver 

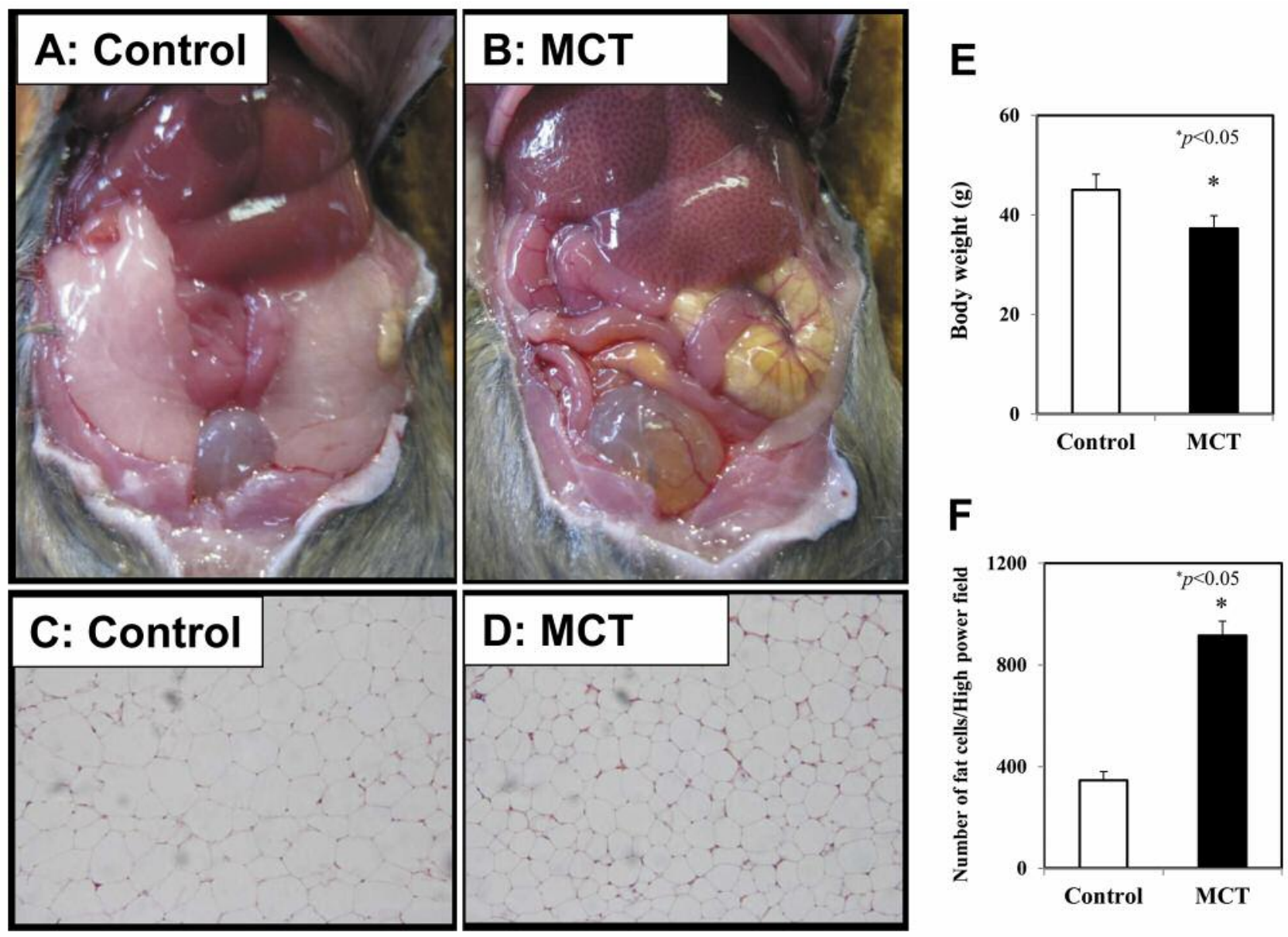

Figure 5. Photographs of intra-abdominal fat and photomicrographs of adipose tissues, body weight after feeding, and numbers of adipocytes. Representative photographs of intra-abdominal fat (A: control group, B: MCT group), and representative photomicroscopes of adipose tissue (C: control group, D: MCT group) are shown. E: body weight, and F: numbers of adipocytes/high power fields are shown. Data represent means \pm SEM. $* p<0.05$ compared to the control group by the unpaired Student's t-test. MCT: Medium-chain triglycerides.

were significantly suppressed in the MCT administration group compared with the control group, despite food intake being the same (Figure 5) (9). Furthermore, MCTs prevented the hypertrophy of adipocytes, leading to a decrease in intraperitoneal adipose tissue mass (Figure 5) (10). These results supported the hypothesis that MCTs may inhibit obesity-related tumor growth. It was reported that adipocytokines play an important role in improving fatty liver, reduction of liver injury, and suppression of hepatic fibrosis (11). It was also reported that adipocytokines are involved in tumor growth $(12,13)$. Importantly, MCTs significantly increase the expression of adipocytokines (10). Indeed, in this study, MCTs significantly decreased these cytokines (Figure 7). Taken together, MCTs had antiinflammatory effects on adipocytokines, most likely leading to the inhibition of tumor growth after DEN administration.
It was reported that ketone bodies suppress tumor growth (14). MCTs are metabolized to ketone bodies more rapidly than other fatty acids (15). In this study, the plasma concentration of ketone body $\beta$-HB significantly increased in the MCT group, compared with the control group (Figure 7). Furthermore, proliferation of MH 134 cells cocultured with $\beta$-HB was significantly inhibited, in a dose dependent manner (Figure 7). Since tumor cells mainly use glucose as their energy source for proliferation, this ketone body derived from MCTs directly inhibited the growth of tumor cells induced by DEN.

\section{Conclusion}

In this study, MCTs decreased inflammation in animals treated with DEN, and increased the expression of 
A

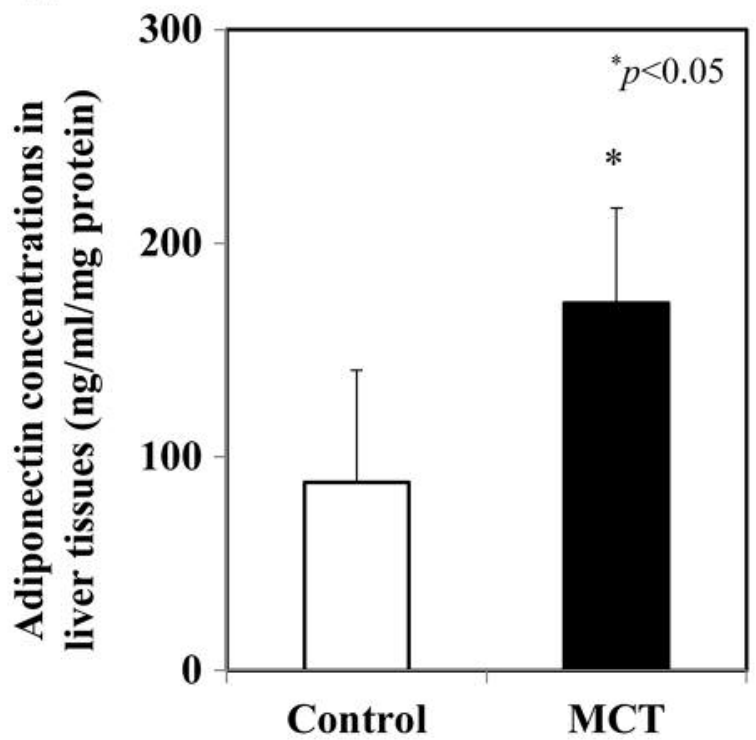

B

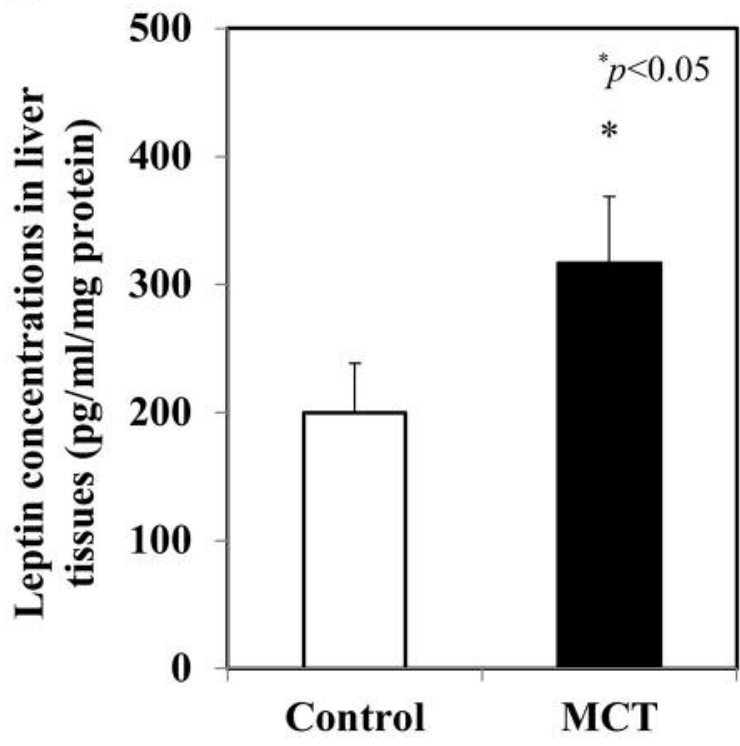

Figure 6. Expression of adipocytokines in liver tissues. A: Adiponectin, and B: leptin. Data represent the mean \pm SEM. * $<<0.05$ compared with the control group by the unpaired Student's t-test. MCT: Medium-chain triglycerides.

A

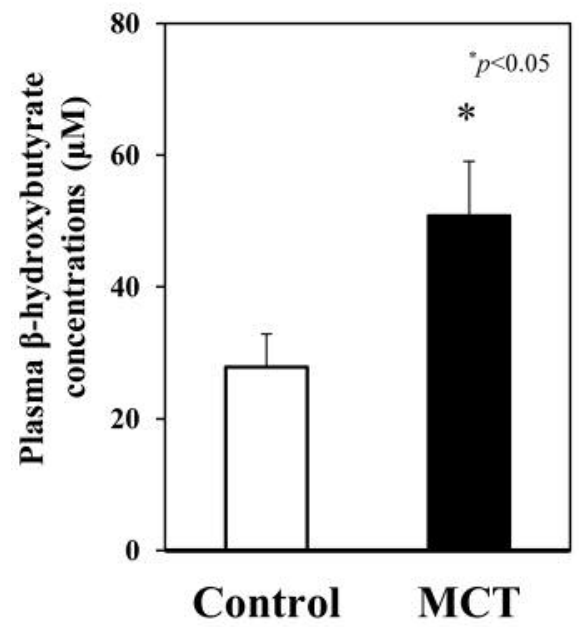

B

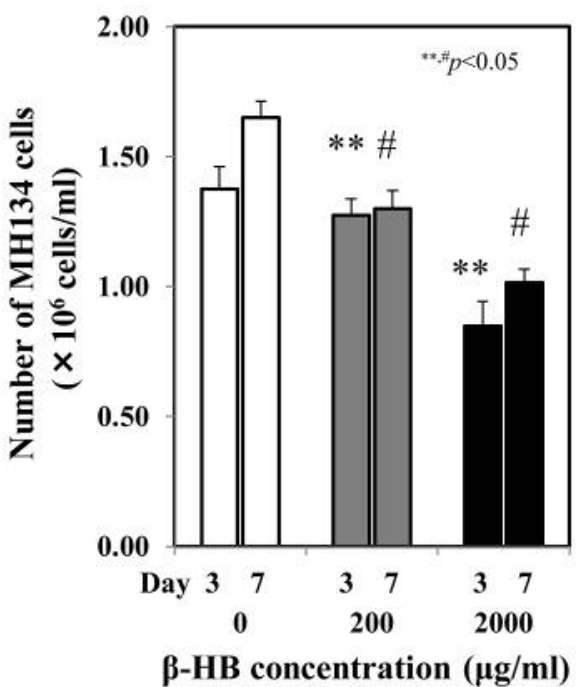

C

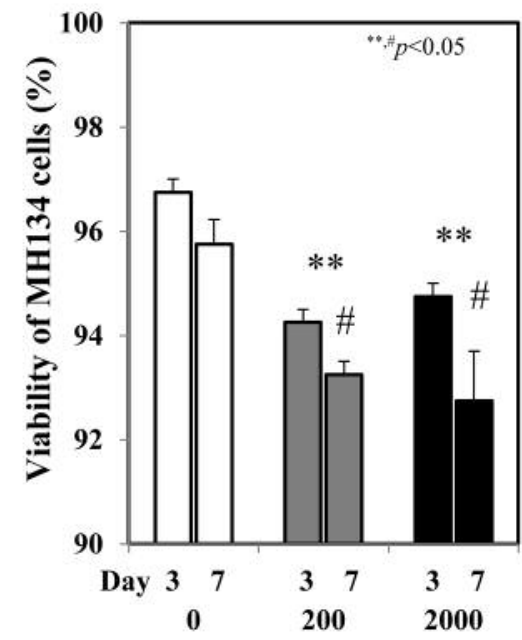

$\beta$-HB concentration $(\mu \mathrm{g} / \mathrm{ml})$

Figure 7. Plasma $\beta$-hydroxybutyrate concentrations, and the number and viability of MF134 cells after incubation. A: Plasma $\beta$-hydroxybutyrate concentrations. B: number of MH134 cells. C: Viability of MH134 cells after incubation. Data represent means \pm SEM. *p $<0.05$ compared to the control group. ${ }^{* *} p<0.05$ compared to cells incubated without $\beta-H B$ at day $3 ;{ }^{\#} p<0.05$ compared with cell incubated without $\beta$-HB at day 7 by the unpaired Student's t-test. MCT: Medium-chain triglycerides, HB: hydroxybutyrate.

adipocytokines in adipose tissue. Moreover, ketone body $\beta$ $\mathrm{HB}$, which is a main metabolite of MCTs, inhibited tumor cell proliferation in vitro. Based on these results, MCTs most likely inhibit the progression of hepatic tumors chemically induced by DEN. Taken together, oral supplement of MCTs or diets containing MCTs could be useful in therapies for hepatic carcinogenesis induced by inflammation caused by viral infection, fatty liver due to metabolic syndrome, alcoholic liver disease, and nonalcohol fatty liver disease. 


\section{Conflicts of Interest}

The Authors declare that they have no conflicts of interest.

\section{Authors' Contributions}

Hiroyuki Wakana contributed to data collection and writing of the paper; Hiroshi Kono contributed to the conception and design of the study; Hisataka Fukushima and Yuuki Nakata contributed to the critical revision of the article; Yoshihiro Akazawa contributed to the analysis and interpretation of the study; Suguru Maruyama and Kotaro Hagio contributed to data collection; Hideki Fujii and Daisuke Ichikawa contributed to the conception and design of this study, and obtained funding.

\section{References}

1 Kitagawa M, Haji S and Amagai T: Elevated serum aa/epa ratio as a predictor of skeletal muscle depletion in cachexic patients with advanced gastro-intestinal cancers. In Vivo 31(5): 10031009, 2017. PMID: 28882973.

2 Bach AC, Ingenbleek Y and Frey A: The usefulness of dietary medium-chain triglycerides in body weight control: Fact or fancy? J Lipid Res 37(4): 708-726, 1996. PMID: 8732772.

3 Grimble RF: Immunonutrition. Curr Opin Gastroenterol 21(2): 216-222, 2005. PMID: 15711216.

4 Lu NT, Liu NM, Vu JQ, Patel D, Cohn W, Capri J, Ziegler M, Patel N, Tramontano A, Williams R, Whitelegge J and French SW: Phospho-network analysis identifies and quantifies hepatitis c virus (hcv)-induced hepatocellular carcinoma (hcc) proteins regulating viral-mediated tumor growth. Cancer Genomics Proteomics 13(5): 339-357, 2016. PMID: 27566653.

5 Kono H, Enomoto N, Connor HD, Wheeler MD, Bradford BU, Rivera CA, Kadiiska MB, Mason RP and Thurman RG: Medium-chain triglycerides inhibit free radical formation and tnf-alpha production in rats given enteral ethanol. Am J Physiol Gastrointest Liver Physiol 278(3): G467-G476, 2000. PMID: 10712267. DOI: 10.1152/ajpgi.2000.278.3.G467

6 Kono H, Fujii H, Asakawa M, Yamamoto M, Matsuda M, Maki A and Matsumoto Y: Protective effects of medium-chain triglycerides on the liver and gut in rats administered endotoxin. Ann Surg 237(2): 246-255, 2003. PMID: 12560783. DOI: 10.1097/01.SLA.0000048450.44868.B1
7 Kono H, Fujii H, Asakawa M, Maki A, Amemiya H, Hirai Y, Matsuda M and Yamamoto M: Medium-chain triglycerides enhance secretory iga expression in rat intestine after administration of endotoxin. Am J Physiol Gastrointest Liver Physiol 286(6): G1081G1089, 2004. PMID: 15132951. DOI: 10.1152/ajpgi.00457.2003

8 Tsuchiya M, Kono H, Matsuda M, Fujii $\mathrm{H}$ and Rusyn I: Protective effect of juzen-taiho-to on hepatocarcinogenesis is mediated through the inhibition of kupffer cell-induced oxidative stress. Int J Cancer 123(11): 2503-2511, 2008. PMID: 18785209. DOI: $10.1002 / \mathrm{ijc} .23828$

9 St-Onge MP, Mayrsohn B, O'Keeffe M, Kissileff HR, Choudhury AR and Laferrere B: Impact of medium and long chain triglycerides consumption on appetite and food intake in overweight men. Eur J Clin Nutr 68(10): 1134-1140, 2014. PMID: 25074387. DOI: 10.1038/ejcn.2014.145

10 Han J, Hamilton JA, Kirkland JL, Corkey BE and Guo W: Medium-chain oil reduces fat mass and down-regulates expression of adipogenic genes in rats. Obes Res 11(6): 734-744, 2003. PMID: 12805394. DOI: $10.1038 /$ oby.2003.103

11 Marra F and Bertolani C: Adipokines in liver diseases. Hepatology 50(3): 957-969, 2009. PMID: 19585655. DOI: 10.1002/hep.23046

12 Yoon KW, Park SY, Kim JY, Lee SM, Park CH, Cho SB, Lee WS, Joo YE, Lee JH, Kim HS, Choi SK and Rew JS: Leptininduced adhesion and invasion in colorectal cancer cell lines. Oncol Rep 31(6): 2493-2498, 2014. PMID: 24700392. DOI: 10.3892/or.2014.3128

13 Hori M, Mutoh M, Ishigamori R, Imai T and Takahashi M: Activated ductal proliferation induced by $n$-nitrosobis (2oxopropyl)amine in fat-infiltrated pancreas of kk-a(y) mice. In Vivo 32(3): 499-505, 2018. PMID: 29695552.

14 Seyfried TN, Flores RE, Poff AM and D'Agostino DP: Cancer as a metabolic disease: Implications for novel therapeutics. Carcinogenesis 35(3): 515-527, 2014. PMID: 24343361. DOI: $10.1093 /$ carcin/bgt480

15 Harvey C, Schofield GM and Williden M: The use of nutritional supplements to induce ketosis and reduce symptoms associated with keto-induction: A narrative review. PeerJ 6: e4488, 2018. PMID: 29576959. DOI: $10.7717 /$ peerj.4488

Received October 16, 2019

Revised November 8, 2019 Accepted November 11, 2019 